\title{
The Role of the Gut Microbiome in Colorectal Cancer
}

\author{
Grace Y. Chen, MD, $\mathrm{PhD}^{1}$ \\ 1 Division of Hematology and Oncology, Department of Internal \\ Medicine, University of Michigan, Ann Arbor, Michigan \\ Clin Colon Rectal Surg 2018;31:192-198.
}

\begin{abstract}
Address for correspondence Grace Y. Chen, MD, PhD, Division of Hematology and Oncology, Department of Internal Medicine, University of Michigan, Ann Arbor, MI (e-mail: gchenry@umich.edu).
\end{abstract}

\begin{abstract}
Keywords

- dysbiosis

- inflammation

- oncomicrobes
\end{abstract}

The gut microbiota is a complex community of microbes that number over $10^{14}$ cells, consisting of bacteria, fungi, protozoa, viruses, and bacteriophage, which reside within the gut and, for the most part, is considered to exist in a symbiotic relationship with the host. Commensal bacteria, for example, have long been recognized to perform vital functions including the ability to metabolize nutrients, vitamins, and drugs; detoxify chemicals; and resist colonization with pathogens. With the advent of culture-independent methods of identifying bacterial populations within the gut, huge advancements have been made in our understanding of the role of the gut microbiota in intestinal health and disease. Importantly, a paradigm has emerged in which intestinal health is maintained and promoted by a "healthy" community of commensal bacteria, whereas perturbations in the microbial community from this healthy, balanced state-a condition known as dysbiosis-results in intestinal disease, including colorectal cancer (CRC). In this article, we will review mouse studies that have provided insight into the mechanisms by which the gut microbiota can both protect against and promote colon tumorigenesis depending on the context, and human studies that have supported the presence of dysbiosis and tumor-promoting bacteria in patients with CRC.

\section{Impact of the Gut Microbiota on Intestinal Homeostasis: Lessons Learned from Mice}

Evidence that the gut microbiota may be important for protecting against the development of colon tumors came from studies of mice deficient in immune receptors that sense bacteria using a mouse model of inflammation-induced colon tumorigenesis in which mice are exposed to the carcinogen azoxymethane (AOM) followed by the administration of water containing dextran sulfate sodium (DSS), which injures the epithelium and induces inflammation. In this model, mice deficient in various Nod-like receptors (NLRs), a family of innate immune receptors that are involved in the recognition of microbial products as well as damage signals, developed significantly more and larger tumors
Issue Theme Translational Research in Colorectal Cancer; Guest Editor: Karin M. Hardiman, MD, PhD

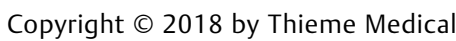
Publishers, Inc., 333 Seventh Avenue, New York, NY 10001, USA. Tel: +1(212) 584-4662. ISSN 1531-0043. 
compared with that in wild-type mice, suggesting that commensal bacteria can limit inflammation-associated colon tumorigenesis by activating these pathways. ${ }^{1-6}$ Consistently, germfree mice that are devoid of all microbes also develop more tumors compared with conventionally housed mice after treatment with AOM and DSS. ${ }^{7}$ Mechanisms behind this protection include (1) promotion of epithelial repair and barrier function that permits resolution of inflammation when the epithelium is damaged, ${ }^{1,6,7}$ (2) the negative regulation of inflammatory pathways that can promote tumorigenesis, ${ }^{4,5}$ (3) increased apoptosis of tumor cells, $^{3}$ (3) prevention of dysbiosis, ${ }^{2}$ and (4) upregulation of cytokines such as IL-18 that promotes tissue repair and antitumor responses. ${ }^{6}$

Bacteria can also produce metabolites that have effects on both the epithelium and immune cells. One particular group of metabolites that have gained a lot of attention is the shortchain fatty acids (SCFAs), such as acetate, propionate, and butyrate, which can only be produced by bacterial fermentation of dietary fiber and resistant starches. Butyrate is an energy source for colon epithelial cells and can modulate inflammation, epithelial proliferation, and apoptosis. In addition, it can affect macrophage and leukocyte responses. ${ }^{8}$ Butyrate is recognized by the host receptors GPR109 and GPR43. ${ }^{9}$ Mice that are deficient in GPR109a exhibited increased tumor susceptibility to colon tumorigenesis in two different colon cancer mouse models, further highlighting the potential beneficial effects of bacterial products. The protective effect of butyrate in these models was attributed to its ability to induce anti-inflammatory responses in dendritic cells and macrophages and the differentiation of naïve T cells into anti-inflammatory regulatory T cells. ${ }^{10}$ The production of SCFAs is dependent on the gut microbiota (specifically members of the Firmicutes phylum) and the availability of dietary fiber, which may partly explain the reduced risk of developing CRC associated with high-fiber diets. However, butyrate may also promote epithelial proliferation, which in turn can increase susceptibility to tumorigenesis. Indeed, in a mouse model of colon cancer in which mice harbor mutations in the APC tumor suppressor gene and the DNA mismatch repair gene $\mathrm{MSH} 2$, butyrate stimulated colon epithelial cell proliferation and increased the number of tumors in these mice. ${ }^{11}$ Thus, genetic factors can influence the effects of the microbiota on tumor development.

Other bacterial metabolites have been suggested to be tumor promoting, such as the secondary bile acids. Bile acids are synthesized from cholesterol within the liver and stored in the gallbladder before being released in the small intestine. Deconjugation of bile acids in the gut by bacteria results in the production of secondary bile acids, such as deoxycholic acid (DCA) and lithocholic acid (LCA), which have cytotoxic properties, namely, by damaging cell membranes that lead to the generation of DNA damaging reactive oxygen species (ROS). ${ }^{12}$ Epithelial damage, in turn, can lead to inflammatory responses and epithelial hyperproliferation that predispose to tumorigenesis. ${ }^{13}$ In the Apc Min mouse model of tumorigenesis, in which mice harbor the commonly mutated Apc tumor suppressor gene that occurs in the majority of sporadic human colon cancers, administration of DCA through drinking water resulted in increased tumors associated with increased epithelial cell proliferation and decreased apoptosis within tumors. ${ }^{14}$

The gut microbiota is typically confined to the colonic lumen and separated from the epithelium by a thin mucus layer; however, when the epithelial barrier is breached as occurs with chemically induced epithelial injury or inflammatory bowel disease, the gut microbiota can translocate into the colonic mucosa and precipitate aberrant and chronic inflammation that is conducive to tumorigenesis. The importance of an intact epithelial barrier and resistance to bacterial translocation that can drive protumorigenic and proinflammatory responses is supported by the observation that bacteria is generally absent in normal biopsies, while bacterial concentrations of $10^{3}$ to $10^{5}$ colony-forming units per microliter were detected in $93 \%$ of colon cancer tissues. ${ }^{15}$ Moreover, in mice harboring an Apc mutation in the intestinal epithelium (CPC-APC mice), ${ }^{16}$ adenoma formation is associated with disruption of the epithelial barrier and the presence of bacteria in the colonic tissue, resulting in the upregulation of cytokines, such as IL-6, IL-17, and IL-23 and induction of proinflammatory Th17 cells. ${ }^{17}$ In addition to NLRs, commensal bacteria are also sensed by Toll-like receptors (TLRs); however, activation of TLRs through the downstream adaptor protein MyD88, unlike that of NLRs, has been associated with increased colon tumorigenesis in mice. Consistently, CPC-APC mice deficient in TLR/MyD88 signaling exhibited reduced IL-6 and IL-17 expression as well as tumor numbers. ${ }^{17}$ Similarly, Apc Min mice that are deficient in MyD88 also have decreased colon tumorigenesis compared with Apc Min that have intact TLR signaling. ${ }^{18}$ Besides proinflammatory cytokines, MyD88 also regulated the expression of growth factors, Cox2, and matrix metalloproteases, all of which have been implicated in colon carcinogenesis. $^{18}$

The study of germfree mice enabled the discovery of a critical role for the gut microbiota in immune cell development, which, in turn, can impact susceptibility to intestinal inflammation and carcinogenesis. Notably, germfree mice have defective production of Th17 and regulatory T (Treg) cells. The balance of proinflammatory Th17 responses and anti-inflammatory regulatory $\mathrm{T}$ cells can contribute to the overall inflammatory tone within the intestine that can influence tumor susceptibility. Germfree mice have reduced numbers of Th17 cells, and recolonization with specific bacterial populations, such as segmented filamentous bacteria in mice, or epithelial-adherent bacteria, including a consortium of 20 bacterial strains isolated from a subject with ulcerative colitis, restored Th17 responses within the gut. ${ }^{19,20}$ Although proinflammatory Th17 responses are important for host defense against bacterial infection and epithelial repair in mice, excessive or dysregulated Th17 responses can also induce tumors. The presence of Th17 cells in the tumor microenvironment, for example, has been associated with poor prognosis. ${ }^{21}$ Furthermore, in the Apc Min mouse model of colon cancer, colonization with the 
common human colonic bacterium, enterotoxigenic Bacteroides fragilis (ETBF), was associated with colitis and increased tumorigenesis. $^{22}$

Regulatory T cells development is also impaired in germfree mice, but can be restored by colonization of specific bacterial populations belonging to Clostridia. ${ }^{23,24}$ Although a decrease in the number of anti-inflammatory regulatory $T$ cells has been associated with increased susceptibility to colitis and tumorigenesis, suppressive $T$ cell responses have also been implicated in decreased antitumor immunity and poorer prognosis. ${ }^{25}$ In Apc Min mice colonized with ETBF that increased tumor multiplicity, depletion of Tregs reduced the number of tumors and enhanced IFN $\gamma$ production, which has antitumor activity. ${ }^{26}$ One mechanism identified by which the gut microbiota can promote regulatory $\mathrm{T}$ cell differentiation is through the production of SCFAs. Germfree mice fed with SCFAs, including butyrate, resulted in increases in Treg numbers within the colon lamina propria. ${ }^{27}$ Butyrate, in particular, can inhibit histone deacetylaces, which results in enhancement of histone acetylation of the Foxp3 promoter and increased Treg differentiation. ${ }^{27,28}$ Despite the known suppressive activities of Tregs on antitumor responses, its precise role in CRC progression remains to be determined, as there are also several studies suggesting that high numbers of intratumoral Foxp $3^{+}$Tregs are associated with improved prognosis of CRC patients. ${ }^{29,30}$ A possible explanation for the conflicting data is that the net effect of Tregs may be context dependent; in the presence of antitumor effector cells, suppressive Tregs can be detrimental, whereas in the presence of proinflammatory, protumorigenic commensal-driven Th17 responses, the presence of Tregs becomes protective. ${ }^{29,30}$

Based on the aforementioned mouse studies, the gut microbiota can promote either health or tumor progression through its inflammatory and proliferative effects likely depending on the context. Studies in mice have also strongly suggested that disturbances in the composition of the gut microbiota (i.e., dysbiosis) can result in the enrichment of bacteria capable of activating inflammatory and cell proliferative pathways that lead to tumorigenesis. ${ }^{2,31,32}$ However, more likely, community-wide changes in bacterial composition rather than a change in the abundance of a single population in the bacterial community are responsible for tumor initiation and/or progression. Consistently, in the AOM/DSS mouse model of inflammation-induced colon tumorigenesis, it was demonstrated that the establishment of different bacterial communities with the use of different antibiotic cocktails resulted in similar levels of tumor development, suggesting that not a single bacterial population was responsible for tumor development. ${ }^{33}$ Furthermore, no single bacterial population directly correlated with tumor outcomes. $^{33}$

Both inflammatory and carcinogenic stimuli can cause changes in the composition of the gut microbiota that predispose to tumorigenesis. ${ }^{34}$ The treatment of mice with the carcinogen AOM followed by DSS was associated with dramatic alterations in the microbial community structure, and significant changes in the relative abundances of multiple bacterial populations. ${ }^{34}$ More importantly, these changes directly contributed to tumor susceptibility, as germfree mice that were recolonized with the gut microbiota of tumor-bearing mice developed more tumors compared with germfree mice harboring the microbiota of naïve, healthy mice after treatment with AOM/DSS. ${ }^{34}$ This study was the first to demonstrate that, at least in mice, the composition of the gut microbiota is an important determinant of colon tumor susceptibility. These studies provide strong rationale for identifying microbial signatures that are predictive of colon cancer risk and for understanding how these signatures are acquired.

\section{Evidence of Dysbiosis in Colorectal Cancer Patients}

Most of the bacteria that reside in the gut are strictly anaerobic and cannot be grown in culture. The ability to identify bacteria using culture-independent methods was therefore a huge advance in the microbiome field. The sequencing of the V1-V3 or V3-V5 variable regions of the bacterial 16S ribosomal RNA has become a standard method of identifying bacterial populations, or operational taxonomic units (OTUs), which generally represent bacterial species whose sequences are within $97 \%$ identical to each other. The Human Microbiome Project (HMP) was a National Institutes of Health-supported mission to determine whether there is a "core" microbiome that defines healthy individuals. The HMP was launched in 2008 and 300 healthy individuals participated, allowing the characterization of the microbiome at 15 or 18 body sites including the gut. Two important findings from this study were that no single bacterial taxa was universally present among all individuals and that there was significant variation in microbial composition between individuals. ${ }^{35}$ Thus, no single "core" microbiome could be identified. ${ }^{35}$ However, despite the diversity in the composition of abundances of bacterial populations between individuals, bacteria identified from stool samples can be categorized into four different community types. ${ }^{36}$ In addition, shotgun metagenomic sequencing to categorize the functional potential of bacterial populations present in the gut revealed conservation of bacterial activities despite bacterial species diversity among individuals. ${ }^{35}$

With the characterization of the healthy gut microbiome, there has been a significant movement toward identifying bacterial changes in specific disease states. These types of studies have generally demonstrated that alterations in the gut microbiome are associated with disease, but whether these alterations actually cause disease has been difficult to determine in humans, but demonstrated in mice. ${ }^{34}$ There are several studies that have suggested that dysbiosis is present in patients with CRC, although the specific bacterial populations that are changed have not been consistent between studies, and most of these studies involved small numbers of patients. For example, comparison of bacterial DNA sequences extracted from the stool of $60 \mathrm{CRC}$ patients with 119 subjects with normal colonoscopies revealed differences in the composition of the gut microbiota with increased 
representation by bacteria belonging to Bacteroides and Prevotella in CRC patients. ${ }^{37}$ A separate study also revealed a different fecal microbiome composition in patients with CRC with depletion of butyrate-producing bacteria. ${ }^{38}$ In both these studies, fecal samples were used to identify differences in the gut microbiome between healthy and CRC patients given the ease of collection; however, stool samples usually reflect luminal bacteria rather than bacteria that are epithelial surface-adherent. In monkeys, the stool has been shown to be highly representative of both luminal and mucosal bacteria in the colon, justifying its use as a surrogate for intestinal bacteria ${ }^{39}$; however, studies in humans comparing bacterial content in mucosal tissue versus fecal samples from healthy subjects have still shown significant differences in composition and diversity between biopsy and stool samples. ${ }^{40-42}$ Regardless, at least two studies examining bacterial populations in cancerous versus normal adjacent tissue from subjects with CRC also demonstrated significant differences in the bacterial composition between normal and tumor tissue, suggesting a role also for mucosal-associated microbiota in tumor progression. ${ }^{43,44}$

The observation that the gut microbiome of healthy individuals is significantly different from that of patients with CRC suggests that the microbiota can serve as biomarkers for CRC detection. A recent study that compared stool samples from 30 healthy and CRC patients identified six different OTUs that were associated with CRCs. Furthermore, when combined with the use of other risk factors such as age and body mass index (BMI), the use of these microbial markers significantly improved the ability to distinguish between healthy and cancer patients. ${ }^{45}$ A subsequent larger study of stool samples from 490 patients (120 CRC patients, 198 with adenomas, and 172 with normal colonoscopies) identified 34 OTUs that were differentially abundant between normal and CRC patients and capable of discriminating between the two populations. This signature alone was not as sensitive as the fecal immunohistochemical test (FIT) in detecting CRCs; however, the use of 23 OTUs in combination with FIT significantly improved the sensitivity of FIT alone for the detection of both adenomas and carcinomas. ${ }^{46}$ Interestingly, these OTUs were not all enriched in CRC patients; rather, many of the OTUs were higher in abundance in normal, healthy patients, suggesting that both the loss of protective bacterial populations together with the acquisition of pathogenic bacteria can contribute to CRC development. Similarly, a microbial signature consisting of 22 OTUs that were either enriched or depleted in CRC patients was also identified in a study of 156 participants. ${ }^{47}$ Although the specific OTUs that are capable of discriminating between healthy and CRC patients are not identical among the different studies, some of them were shared, and include bacteria associated with periodontal disease, such as Fusobacterium nucleatum and Peptostreptococcus stomatis, as well as bacteria that are capable of producing butyrate. Thus, it is also possible that specific bacterial activities may be a more important determinant of disease risk than specific OTUs. Additional studies with larger number of subjects may clarify this issue and may also identify additional microbiome-based biomarkers.

\section{The Existence of Oncomicrobes}

There has been significant interest in identifying specific bacteria that directly contribute or trigger the development of CRC and, therefore, can be considered carcinogenic, or an "oncomicrobe." A classic oncomicrobe is the human papillomavirus, the major etiologic agent of cervical cancer worldwide, which can cause transformation of cells through the interaction of the viral proteins E6 and E7 with the tumor suppressor proteins p53 and retinoblastoma, respectively, causing their functional deactivation. ${ }^{48}$ Very few bacteria have been definitely characterized as oncomicrobes, in part, because of the reliance on Koch's postulates to establish causality between a bacterial strain and disease. There are four criteria that comprise Koch's postulates: (1) the pathogen must be increased in abundance in the diseased state, (2) the pathogen must be isolatable and cultivatable, (3) the pathogen when transferred into a healthy organism must recapitulate the diseased state, and (4) the pathogen when re-isolated from the diseased organism must be identical to the original causative agent. The challenge of cultivating the majority of intestinal bacteria and further demonstrating causality has significantly limited our ability to identify true oncomicrobes in CRC.

There has been interest, however, in certain bacterial strains that are potentially oncomicrobes. For example, the polyketide synthase ( $p k s$ ) island positive Escherichia coli was increased in prevalence in colon tissue specimens isolated from CRC patients. ${ }^{49}$ In mice, monocolonization of germfree IL-10 ${ }^{-1-}$ mice, which are prone to developing spontaneous colitis, with $\Delta p k s E$. coli developed significantly fewer tumors compared with mice that harbored the wild-type $p k s^{+} E$. coli. The increase in tumors did not correlate with the severity of inflammation that developed, suggesting a mechanism independent of proinflammatory responses to $E$. coli. Rather, $E$. coli was demonstrated to induce DNA damage in vitro and cause G2/M arrest, ${ }^{49}$ consistent with the known genotoxic properties of the PKS gene product colibactin. ${ }^{49-51}$

One bacterium that has generated significant interest is $F$. nucleatum, which is enriched in subjects with either adenomas or CRC. ${ }^{52}$ F. nucleatum is an oral commensal associated with periodontal disease. In a seminal study in which the microbial composition of nine CRCs was compared with matched normal colons, F. nucleatum was most significantly different in abundance between the two groups. ${ }^{52}$ This study also demonstrated the presence of $F$. nucleatum within colorectal tumors by fluorescence in situ hybridization. F. nucleatum was also subsequently found in increased abundance in stool samples from patients with either adenomas or adenocarcinomas. ${ }^{53}$

The association of this bacterium with CRC was further validated in a larger cohort of 95 paired specimens of colon cancer and normal colon tissue. Furthermore, in a review of the Nurses' Health Study and Health Professionals Follow-up Study consisting of 1,069 rectal and colon cancer cases, the 
presence of $F$. nucleatum positively correlated with proximal tumor location as well as microsatellite instable (MSI) tumors. ${ }^{54}$ More importantly, the presence of $F$. nucleatum was associated with higher CRC-specific mortality, suggesting that $F$. nucleatum can serve as a prognostic biomarker. ${ }^{54}$ The relationship between increased mortality and F. nucleatum may not be direct, as the amount of $F$. nucleatum in tumor tissue was inversely associated with the density of $\mathrm{CD}^{+} \mathrm{T}$ cells, which has been associated with better prognosis. ${ }^{55,56}$

F. nucleatum has several properties that are conducive to tumorigenesis. It is capable of eliciting proinflammatory responses and can adhere as well as invade host epithelial cells. $^{57}$ In fact, RNAseq of 133 colon tumors in the Cancer Genome Atlas identified a Fusobacterium-associated human CRC gene expression signature consisting of proinflammatory and protumorigenic factors including Cox-2, IL-6, and TNF $\alpha{ }^{53}$ Although these observations have only established an association between $F$. nucleatum abundance and CRC in humans, studies on mouse suggest that $F$. nucleatum directly promotes tumor growth. In the Apc Min mouse model, enforced colonization of $\mathrm{F}$. nucleatum resulted in increased tumor multiplicity. In particular, Apc Min mice administered F. nucleatum developed tumors that had increased infiltration of myeloid-derived suppressor cells that can promote tumor progression by inhibiting antitumor responses. ${ }^{53}$ Consistently, F. nucleatum is capable of directly binding to tumor cells and inhibiting NK cell-mediated tumor killing by interacting with the inhibitory NK cell receptor TIGIT. ${ }^{58}$ Besides NK cells, tumor-infiltrating lymphocytes also express TIGIT, and F. nucleatum can inhibit their activity as well. ${ }^{58}$ The protumorigenic activities of $F$. nucleatum are not limited to its immunosuppressive effects, as it can also bind to E-cadherin and activate ßeta-catenin, resulting in increased proliferation of CRC cell lines in vitro and expression of proinflammatory cytokines in xenograft tumors in vivo. F. nucleatum proteins specifically involved in binding to TIGIT and E-cadherin are Fap2 and FadA, respectively, which may provide potential targets for CRC treatment and prevention. It is important to note, however, that $F$. nucleatum is not universally present in CRCs; its DNA was detected in only approximately $13 \%$ of CRC cases, and therefore any targeted treatment will need to be individualized. ${ }^{54}$ As suggested by both mouse and human studies, although there remains significant interest in identifying specific oncomicrobes that can be used as biomarkers or targeted for the prevention and treatment of CRC, it is likely that not one microbe is responsible for colon tumor initiation and/or progression, but a change in the balance of both harmful and protective bacterial populations.

\section{Future Directions in Microbiome Research}

As in healthy individuals, there may be significant interindividual diversity in the gut microbiome of CRC patients. However, it is also likely that, as demonstrated for healthy individuals, the functional potential or activities represented by individual bacterial communities are relatively conserved, ${ }^{35}$ suggesting that it would be important to determine not only bacterial membership but also bacterial activities represented in the gut microbiomes of healthy and CRC patients. Thus, there is increasing interest in identifying differences in the functional potential, transcribed genes, metabolic profiles, and expressed proteins of bacterial communities between healthy and CRC patients by metagenomics, metatranscriptomics, metabolomics, and metaproteomics, respectively. Metagenomic sequencing, for example, of stool samples from CRC patients and normal controls suggested an increase in the functional potential of the microbiota of CRC patients for degradation of host glycans and amino acids as well as lipopolysaccharide metabolism, which can contribute to proinflammatory responses. ${ }^{47}$ Another study investigating global metabolites in stool samples of CRC patients suggested increases in certain amino acids that may be related to microbial degradation of dietary proteins. ${ }^{38}$ However, the significance of these findings and the nature of their connection to the pathogenesis of CRC remain to be determined. Thus far, metaproteomic studies to identify bacterial proteins associated with CRC have not yet been performed, perhaps due in part to the limited availability of databases that encompass the protein diversity of the gut microbiota and the need for complete metagenome sequences for accurate bacterial protein identification. ${ }^{59}$

In addition to understanding how the composition of the gut microbiome modulates CRC susceptibility, there is growing interest in determining whether the composition of the gut microbiota is also predictive of responses to chemotherapy. Two seminal studies revealed that the gut microbiota may be important in chemotherapy-induced antitumor responses. ${ }^{60,61}$ Specifically, germfree or antibiotic-treated mice exhibited decreased responses to chemotherapy. This was attributed to the ability of bacterial products to elicit inflammatory responses in antigen-presenting cells that promote antitumor immunity. Chemotherapy can also alter the composition of the gut microbiota; however, it is not known whether specific bacterial populations directly contribute to treatment response. Recently, it was demonstrated that the presence of Bifidobacterium species is critical for antitumor responses associated with anti-PD-L1 therapy in a mice model of melanoma by enhancing dendritic activation and priming of antitumor $\mathrm{T}$ cell responses in mice. ${ }^{62}$ Whether the gut microbiota influences treatment responses in CRC patients remains to be determined.

The area of microbiome research has exploded in the past several years due to advancing technologies in the identification and analysis of bacteria. Although it is becoming increasingly clear that gut microbiota is an important determinant in promoting health and modulating CRC risk, the precise mechanisms involved still remain to be fully elucidated. Regardless, continued research efforts to identify bacterial populations and activities associated with tumor initiation and progression will be critical for developing microbiome-based strategies for the early detection, prevention, and treatment of CRC. 


\section{References}

1 Chen GY, Shaw MH, Redondo G, Núñez G. The innate immune receptor Nod1 protects the intestine from inflammation-induced tumorigenesis. Cancer Res 2008;68(24):10060-10067

2 Couturier-Maillard A, Secher T, Rehman A, et al. NOD2-mediated dysbiosis predisposes mice to transmissible colitis and colorectal cancer. J Clin Invest 2013;123(02):700-711

3 Hu B, Elinav E, Huber S, et al. Inflammation-induced tumorigenesis in the colon is regulated by caspase- 1 and NLRC4. Proc Natl Acad Sci U S A 2010;107(50):21635-21640

4 Zaki MH, Lamkanfi M, Kanneganti TD. The Nlrp3 inflammasome: contributions to intestinal homeostasis. Trends Immunol 2011; 32(04):171-179

5 Zaki MH, Vogel P, Malireddi RK, et al. The NOD-like receptor NLRP12 attenuates colon inflammation and tumorigenesis. Cancer Cell 2011;20(05):649-660

6 Chen GY, Liu M, Wang F, Bertin J, Núñez G. A functional role for Nlrp6 in intestinal inflammation and tumorigenesis. J Immunol 2011;186(12):7187-7194

7 Zhan Y, Chen PJ, Sadler WD, et al. Gut microbiota protects against gastrointestinal tumorigenesis caused by epithelial injury. Cancer Res 2013;73(24):7199-7210

8 Chang PV, Hao L, Offermanns S, Medzhitov R. The microbial metabolite butyrate regulates intestinal macrophage function via histone deacetylase inhibition. Proc Natl Acad Sci U S A 2014;111(06):2247-2252

9 Ganapathy V, Thangaraju M, Prasad PD, Martin PM, Singh N. Transporters and receptors for short-chain fatty acids as the molecular link between colonic bacteria and the host. Curr Opin Pharmacol 2013;13(06):869-874

10 Singh N, Gurav A, Sivaprakasam S, et al. Activation of Gpr109a, receptor for niacin and the commensal metabolite butyrate, suppresses colonic inflammation and carcinogenesis. Immunity 2014;40(01):128-139

11 Belcheva A, Irrazabal T, Robertson SJ, et al. Gut microbial metabolism drives transformation of MSH2-deficient colon epithelial cells. Cell 2014;158(02):288-299

12 Louis P, Hold GL, Flint HJ. The gut microbiota, bacterial metabolites and colorectal cancer. Nat Rev Microbiol 2014;12(10): 661-672

13 Ochsenkühn T, Bayerdörffer E, Meining A, et al. Colonic mucosal proliferation is related to serum deoxycholic acid levels. Cancer 1999;85(08):1664-1669

14 Cao H, Luo S, Xu M, et al. The secondary bile acid, deoxycholate accelerates intestinal adenoma-adenocarcinoma sequence in Apc $(\mathrm{min} /+)$ mice through enhancing Wnt signaling. Fam Cancer 2014;13(04):563-571

15 Swidsinski A, Khilkin M, Kerjaschki D, et al. Association between intraepithelial Escherichia coli and colorectal cancer. Gastroenterology 1998;115(02):281-286

16 Hinoi T, Akyol A, Theisen BK, et al. Mouse model of colonic adenoma-carcinoma progression based on somatic Apc inactivation. Cancer Res 2007;67(20):9721-9730

17 Grivennikov SI, Wang K, Mucida D, et al. Adenoma-linked barrier defects and microbial products drive IL-23/IL-17-mediated tumour growth. Nature 2012;491(7423):254-258

18 Rakoff-Nahoum S, Medzhitov R. Regulation of spontaneous intestinal tumorigenesis through the adaptor protein MyD88. Science 2007;317(5834):124-127

19 Ivanov II, Atarashi K, Manel N, et al. Induction of intestinal Th17 cells by segmented filamentous bacteria. Cell 2009;139(03): 485-498

20 Atarashi $\mathrm{K}$, Tanoue $\mathrm{T}$, Ando $\mathrm{M}$, et al. Th17 cell induction by adhesion of microbes to intestinal epithelial cells. Cell 2015; 163(02):367-380

21 Tosolini M, Kirilovsky A, Mlecnik B, et al. Clinical impact of different classes of infiltrating T cytotoxic and helper cells (Th1, th2, treg, th17) in patients with colorectal cancer. Cancer Res 2011;71(04):1263-1271

$22 \mathrm{Wu} \mathrm{S}$, Rhee KJ, Albesiano E, et al. A human colonic commensal promotes colon tumorigenesis via activation of T helper type $17 \mathrm{~T}$ cell responses. Nat Med 2009;15(09):1016-1022

23 Atarashi K, Tanoue T, Oshima K, et al. Treg induction by a rationally selected mixture of Clostridia strains from the human microbiota. Nature 2013;500(7461):232-236

24 Atarashi K, Tanoue T, Shima T, et al. Induction of colonic regulatory T cells by indigenous Clostridium species. Science 2011;331 (6015):337-341

25 Terme M, Pernot S, Marcheteau E, et al. VEGFA-VEGFR pathway blockade inhibits tumor-induced regulatory T-cell proliferation in colorectal cancer. Cancer Res 2013;73(02):539-549

26 Geis AL, Fan H, Wu X, et al. Regulatory T-cell response to Enterotoxigenic Bacteroides fragilis colonization triggers IL17-dependent colon carcinogenesis. Cancer Discov 2015;5(10):1098-1109

27 Smith PM, Howitt MR, Panikov N, et al. The microbial metabolites, short-chain fatty acids, regulate colonic Treg cell homeostasis. Science 2013;341(6145):569-573

28 Furusawa Y, Obata Y, Fukuda S, et al. Commensal microbe-derived butyrate induces the differentiation of colonic regulatory $\mathrm{T}$ cells. Nature 2013;504(7480):446-450

29 Zhang X, Kelaria S, Kerstetter J, Wang J. The functional and prognostic implications of regulatory $\mathrm{T}$ cells in colorectal carcinoma. J Gastrointest Oncol 2015;6(03):307-313

30 Ladoire S, Martin F, Ghiringhelli F. Prognostic role of FOXP3+ regulatory $T$ cells infiltrating human carcinomas: the paradox of colorectal cancer. Cancer Immunol Immunother 2011;60(07):909-918

31 Man SM, Zhu Q, Zhu L, et al. Critical role for the DNA sensor AIM2 in stem cell proliferation and cancer. Cell 2015;162(01):45-58

$32 \mathrm{Hu} \mathrm{B}$, Elinav E, Huber S, et al. Microbiota-induced activation of epithelial IL-6 signaling links inflammasome-driven inflammation with transmissible cancer. Proc Natl Acad Sci U S A 2013; 110(24):9862-9867 [Corrected in "Proc Natl Acad Sci U S A 2013;110(31):12852" (Note: Wunderlich, Claudia [added]; Wunderlich, Thomas [added])]

33 Zackular JP, Baxter N, Chen GY, Schloss PD. Manipulation of the gut microbiota reveals role in colon tumorigenesis. mSphere 2015;1(01):e00001-e00015

34 Zackular JP, Baxter NT, Iverson KD, et al. The gut microbiome modulates colon tumorigenesis. MBio 2013;4(06):e00692-e13

35 Human Microbiome Project Consortium. Structure, function and diversity of the healthy human microbiome. Nature 2012;486 (7402):207-214

36 Ding T, Schloss PD. Dynamics and associations of microbial community types across the human body. Nature 2014;509 (7500):357-360

37 Sobhani I, Tap J, Roudot-Thoraval F, et al. Microbial dysbiosis in colorectal cancer (CRC) patients. PLoS One 2011;6(01):e16393

38 Weir TL, Manter DK, Shefl in AM, Barnett BA, Heuberger AL, Ryan EP. Stool microbiome and metabolome differences between colorectal cancer patients and healthy adults. PLoS One 2013;8(08):e70803

39 Yasuda K, Oh K, Ren B, et al. Biogeography of the intestinal mucosal and lumenal microbiome in the rhesus macaque. Cell Host Microbe 2015;17(03):385-391

40 Momozawa Y, Deffontaine V, Louis E, Medrano JF. Characterization of bacteria in biopsies of colon and stools by high throughput sequencing of the $\mathrm{V} 2$ region of bacterial 16S rRNA gene in human. PLoS One 2011;6(02):e16952

41 Durbán A, Abellán JJ, Jiménez-Hernández N, et al. Assessing gut microbial diversity from feces and rectal mucosa. Microb Ecol 2011;61(01):123-133

42 Eckburg PB, Bik EM, Bernstein CN, et al. Diversity of the human intestinal microbial flora. Science 2005;308(5728):635-1638

43 Gao Z, Guo B, Gao R, Zhu Q, Qin H. Microbiota disbiosis is associated with colorectal cancer. Front Microbiol 2015;6:20 
44 Marchesi JR, Dutilh BE, Hall N, et al. Towards the human colorectal cancer microbiome. PLoS One 2011;6(05):e20447

45 Zackular JP, Rogers MA, Ruffin MT IV, Schloss PD. The human gut microbiome as a screening tool for colorectal cancer. Cancer Prev Res (Phila) 2014;7(11):1112-1121

46 Baxter NT, Ruffin MT IV, Rogers MA, Schloss PD. Microbiota-based model improves the sensitivity of fecal immunochemical test for detecting colonic lesions. Genome Med 2016;8(01):37

47 Zeller G, Tap J, Voigt AY, et al. Potential of fecal microbiota for earlystage detection of colorectal cancer. Mol Syst Biol 2014;10:766

48 Münger K, Scheffner M, Huibregtse JM, Howley PM. Interactions of HPV E6 and E7 oncoproteins with tumour suppressor gene products. Cancer Surv 1992;12:197-217

49 Arthur JC, Perez-Chanona E, Mühlbauer M, et al. Intestinal inflammation targets cancer-inducing activity of the microbiota. Science 2012;338(6103):120-123

50 Nougayrède JP, Homburg S, Taieb F, et al. Escherichia coli induces DNA double-strand breaks in eukaryotic cells. Science 2006;313 (5788):848-851

51 Cuevas-Ramos G, Petit CR, Marcq I, Boury M, Oswald E, Nougayrède JP. Escherichia coli induces DNA damage in vivo and triggers genomic instability in mammalian cells. Proc Natl Acad Sci U S A 2010;107(25):11537-11542

52 Kostic AD, Gevers D, Pedamallu CS, et al. Genomic analysis identifies association of Fusobacterium with colorectal carcinoma. Genome Res 2012;22(02):292-298

53 Kostic AD, Chun E, Robertson L, et al. Fusobacterium nucleatum potentiates intestinal tumorigenesis and modulates the tumorimmune microenvironment. Cell Host Microbe 2013;14(02): 207-215
54 Mima K, Nishihara R, Qian ZR, et al. Fusobacterium nucleatum in colorectal carcinoma tissue and patient prognosis. Gut 2015; (Aug):26

55 Mima K, Sukawa Y, Nishihara R, et al. Fusobacterium nucleatum and T Cells in Colorectal Carcinoma. JAMA Oncol 2015;1(05): 653-661

56 Galon J, Costes A, Sanchez-Cabo F, et al. Type, density, and location of immune cells within human colorectal tumors predict clinical outcome. Science 2006;313(5795):1960-1964

57 Bachrach G, Ianculovici C, Naor R, Weiss EI. Fluorescence based measurements of Fusobacterium nucleatum coaggregation and of fusobacterial attachment to mammalian cells. FEMS Microbiol Lett 2005;248(02):235-240

58 Gur C, Ibrahim Y, Isaacson B, et al. Binding of the Fap2 protein of Fusobacterium nucleatum to human inhibitory receptor TIGIT protects tumors from immune cell attack. Immunity 42(02): 2015;344-355

59 Hettich RL, Pan C, Chourey K, Giannone RJ. Metaproteomics: harnessing the power of high performance mass spectrometry to identify the suite of proteins that control metabolic activities in microbial communities. Anal Chem 2013;85(09):4203-4214

60 Iida N, Dzutsev A, Stewart CA, et al. Commensal bacteria control cancer response to therapy by modulating the tumor microenvironment. Science 2013;342(6161):967-970

61 Viaud S, Saccheri F, Mignot G, et al. The intestinal microbiota modulates the anticancer immune effects of cyclophosphamide. Science 2013;342(6161):971-976

62 Sivan A, Corrales L, Hubert N, et al. Commensal Bifidobacterium promotes antitumor immunity and facilitates anti-PD-L1 efficacy. Science 2015;350(6264):1084-1089 